\title{
Milstein, Alexander (2016): Territorialer Zusammenhalt und Daseinsvorsorge - Grundlagen des europäischen Raumentwicklungsrechts
}

\author{
Berlin: Lexxion Verlag. = Zentralinstitut für Raumplanung an der Universität Münster, Beiträge \\ zum Raumplanungsrecht 253.580 S.
}

\author{
Matthias Kraus ${ }^{1}$ \\ Online publiziert: 12. Februar 2018 \\ (c) Springer-Verlag GmbH Deutschland, ein Teil von Springer Nature 2018
}

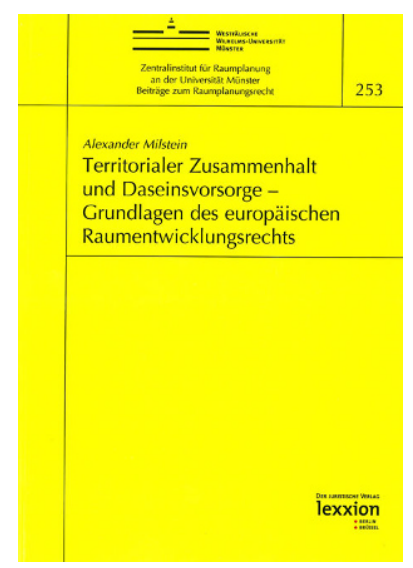

Mit dem Inkrafttreten des Reformvertrages von Lissabon im Dezember 2009 ist das Ziel des territorialen Zusammenhalts auch primärrechtlich neben die Ziele der ökonomischen und sozialen Kohäsion getreten. Seine Herausbildung war nicht zuletzt das Resultat eines langen Prozesses einer in mitgliedschaftlicher Zusammenarbeit praktizierten Raumentwicklungspolitik. Auch wenn unbestritten ist, dass die räumliche Vielfalt der Europäischen Union (EU), die starken wirtschaftlichen Disparitäten und unterschiedliche regionale Entwicklungsperspektiven nach einer räumlichen Betrachtung und Koordinierung der diversen Entwicklungsansätze und Politiken auf europäischer Ebene verlangen, besteht bisher weder ein einheitliches Verständnis der mit der territorialen Kohäsion verbundenen Kompetenzen, noch

Dr. Matthias Kraus

matthias.kraus@reg-ob.bayern.de

1 Regierung von Oberbayern,

Maximilianstraße 39, 80538 München,

Deutschland ist geklärt, wie sich diese zu den nationalstaatlichen Raumentwicklungskompetenzen verhalten.

Im vorliegenden Werk, das im Wintersemester 2015/2016 von der Rechtswissenschaftlichen Fakultät der Westfälischen Wilhelms-Universität Münster als Dissertation angenommen wurde, wird als breit angelegte Untersuchung der Versuch unternommen, das Konzept des territorialen Zusammenhalts insbesondere im Kontext wichtiger EUPolitiken $\mathrm{zu}$ erfassen und mit dem inhaltlichen Blick auf die Planung der öffentlichen Versorgung die Grundlagen eines europäischen Raumentwicklungsrechts $\mathrm{zu}$ destillieren. Mit ihrem die Rechtsordnungen und Fachpolitiken übergreifenden Betrachtungsansatz zeichnet die Arbeit ein ,großes Bild' und sticht damit aus den einschlägigen Veröffentlichungen heraus. Sie veranschaulicht eindrücklich, wie zunehmend querschnittsbezogene räumliche Aspekte in verschiedenen europäischen Politiken Wirkung entfalten. Die thematische Spanne bedingt zugleich, dass nicht alle offenen oder umstrittenen rechtlichen Fragen des Themenkomplexes ausführlich gewürdigt werden können. Hier bietet sie ergänzend umfangreiche Verweise auf weiterführende Literatur und Rechtsprechung.

Nach einem einführenden ersten Teil steht im Zentrum des zweiten Teils zunächst eine rechtsvergleichende Untersuchung: Die deutsche „Raumordnung“ (Kapitel § 2) und das französische „Aménagement du territoire“ (Kapitel § 3) stehen sich als zwei nationale Ausprägungen von ,Raumentwicklungsansätzen ' gegenüber, die sich in ihrem Steuerungsanspruch und ihrem Instrumentarium stark unterscheiden. Für beide Rechtssysteme beschreibt Milstein die verfassungsrechtlichen Fundamente, die die jeweilige nationale Raumentwicklungsplanung tragen, die historische Entwicklung, das Planungsverständnis und die Planungsebenen in beiden Staaten. Er veranschaulicht die unter- 
schiedlichen Ansätze zur Gewährleistung der öffentlichen Versorgung und belegt, wie die Raumordnung in Deutschland als überfachliche Koordinierungsplanung wenig eigenen Einfluss auf die Verwirklichung der Daseinsvorsorge hat. Demgegenüber erweist sich das auf regionalökonomischen Ansätzen aufbauende „Aménagement du territoire“ als vornehmlich verwaltungsinterner Planungsansatz stärker verwirklichungsorientiert.

In die Betrachtung des Unionsrechts steigt Milstein in Kapitel $\S 4$ mit einer Untersuchung des in Art. 3 Abs. 3 UAbs. 3 EUV $^{1}$ verankerten Kohärenzziels in seiner territorialen Dimension ein. Deren materiellem Gehalt nähert er sich zunächst über den integrationstheoretischen Hintergrund, den er ausgehend von den Beispielen der französischen und deutschen Verfassungstradition erörtert. Anschließend führt er aus, wie sich die Europäische Union als Rechts- und Solidargemeinschaft versteht und beleuchtet die unterschiedlichen Ausprägungen des Solidaritätsprinzips. Zur Begründung des Bedeutungsgehalts der europäischen Kohäsion verweist er zunächst auf den Binnenmarkt und das diesen tragende Wettbewerbsprinzip. Als normative Vorgabe für die Entwicklung des europäischen Territoriums leitet er aus dem Kohärenzziel ein polyzentrisches Raummodell ab. Abschließend diskutiert er in diesem Kapitel die Rechtswirkungen des Kohärenzziels, das als final ausgerichtetes Integrationsprogramm unmittelbar nur die Organe der Union verpflichte.

In Kapitel $\S 5$ wird belegt, wie in der europäischen Wettbewerbspolitik in Bezug auf die Dienste von allgemeinem wirtschaftlichem Interesse schon in den 1990er-Jahren den Interessen des territorialen Zusammenhalts Rechnung getragen wurde. Anhand der historischen Entwicklung der europäischen Wirtschaftsverfassung wird gezeigt, wie die Marktöffnung Veränderungen für verschiedene Bereiche der öffentlichen Versorgung gebracht hat. Hierzu legt die Arbeit in einem Überblick die wichtigsten wettbewerbsrechtlichen Vorschriften für öffentliche Unternehmen und Mitgliedstaaten und ihre Bedeutung für die öffentliche Versorgung dar, um im Anschluss die Wirkungen der an die Mitgliedstaaten gerichteten Beihilfevorschriften auf diesem Feld zu skizzieren. Milstein zeigt, wie insbesondere durch die Rechtsprechung des Europäischen Gerichtshofes das Institut der ,Dienste von allgemeinem wirtschaftlichen Interesse" als Gegenpol zum Primat des Wettbewerbs entwickelt wurde und damit für diesen Bereich - als Ausnahme von der Geltung des Wettbewerbsrechts - den Mitgliedstaaten unter anderem Eingriffsmöglichkeiten in den Markt eröffnet sind, um den territorialen Zusammenhalt zu fördern. In diesem Zusammenhang diskutiert er auch die rechtliche Qualität der Verankerung des Zugangs zu Dienstleistungen von allgemeinem wirtschaftlichem Interesse in

\footnotetext{
1 Vertrag über die Europäische Union.
}

Art. $14 \mathrm{AEUV}^{2}$ und Art. $36 \mathrm{GRCH}^{3}$, um zu dem Schluss zu kommen, dass diesen Normen kein über den bisherigen Rechtsstand hinausgehender Gehalt zukommt.

Kapitel $\S 6$ widmet sich der Europäischen Kohäsionspolitik, die - stark am französischen Raumentwicklungsmodell des „Aménagement du territoire“ orientiert - über ein fondsbasiertes Modell die Beförderung der Entwicklungsvorstellungen stützt. Die Darstellung zeigt die historische Entwicklung der Kohäsionspolitik zu einem eigenständigen Politikbereich innerhalb der EU-Verträge, der mit dem Vertrag von Lissabon um die räumliche Dimension erweitert wurde. Sie erläutert die einschlägigen Zielvorgaben und Kompetenzen der Union und stellt die drei Fonds der Kohäsionspolitik und ihren Beitrag zum territorialen $\mathrm{Zu}$ sammenhalt vor. Den Schwerpunkt setzt die Arbeit auf die Beschreibung der sekundärrechtlich normierten mehrstufigen Programmplanung der Kohäsionspolitik. Sie zeigt, dass insbesondere die Programmplanung im engeren Sinne die räumliche Koordinierung mit den Raumakteuren der verschiedenen Ebenen trägt. Den Abschluss dieses Kapitels bildet die Behandlung des organisationsrechtlichen Instruments des „Europäischen Verbund für territoriale Zusammenarbeit“. Die im Schrifttum aufgeworfenen Bedenken hinsichtlich der kompetenzrechtlichen Grundlagen dieses Instruments teilt Milstein nicht und sieht es von Art. 175 Abs. 3 AEUV gedeckt.

Ein funktionsfähiges und lückenloses transeuropäisches Infrastrukturnetz ist wesentliche Voraussetzung für die Förderung des territorialen Zusammenhalts und die Gewährleistung von Diensten von allgemeinem Interesse. Der Abschnitt über die Transeuropäischen Netze in Kapitel $\S 7$ zeichnet zunächst einen Überblick über die historische Entwicklung der transeuropäischen Infrastrukturpolitik und das Infrastrukturkonzept der Union gemäß Art. 170 AEUV. Dazu erörtert Milstein kurz die Frage der Kompetenz der Union auf diesem Gebiet und ihre Abgrenzung zu anderen Politikbereichen. Als zentrale Instrumente einer europäischen „Netzplanung“ erörtert Milstein die auf der Grundlage von Art. 171 Abs. 1 AEUV aufgestellten sekundärrechtlichen Leitlinien für Vorhaben von gemeinsamem Interesse, beschreibt ihren Regelungsgehalt, das Zustandekommen und ihre Rechtsnatur. Zu der Frage nach der Reichweite der Vorgaben vertritt er - entgegen dem wohl überwiegenden Schrifttum - die These, dass eine weitreichende Verwirklichungspflicht für Mitgliedstaaten besteht. Diese Auffassung führt folgerichtig dazu, dass Milstein den Leitlinien erheblichen Einfluss auf die nationale Infrastrukturplanung einräumt und sich demnach die Bedarfsplanung des Bundes eng an den europäischen Leitlinien orientieren müsste.

\footnotetext{
2 Vertrag über die Arbeitsweise der Europäischen Union.

${ }_{3}$ Charta der Grundrechte der Europäischen Union.
} 
In Kapitel $\S 8$ schließlich ordnet Milstein das Konzept der „Europäischen Territorialen Governance“ als Baustein eines europäischen Raumentwicklungsrechts ein. Er verweist auf die Abkehr vom Prinzip der hoheitlichen Planung und den Wechsel im Planungsverständnis, das auch auf europäischer Ebene zunehmend auf kooperative Instrumente unter Einbindung von Akteuren aus Verwaltung, Privatwirtschaft und Zivilgesellschaft setzt. Im Zentrum seiner Betrachtung stehen mit dem Europäischen Raumentwicklungskonzept EUREK, den Territorialen Agenden und den Makroregionalen Strategien Ansätze, die außerhalb der primärrechtlich verankerten Politikbereiche die Raumentwicklung in Europa beeinflussen. Diese fasst Milstein unter dem Begriff der „europäischen Raumentwicklungsdokumente“ als Handlungsform zusammen und ordnet sie aufgrund ihrer geringen rechtlichen Verbindlichkeit bei gleichzeitig großer tatsächlicher Bedeutung der rechtsdogmatischen Kategorie des faktischen Rechts (,Soft-laws“) zu. In Bezug auf die Kompetenz und Legitimation für diese raumbezogenen Maßnahmen verweist er auf eine in Art. 171 Abs. 2 S. 2
AEUV verortete Koordinationskompetenz der Union. Für das Verhältnis zur mitgliedschaftlichen Raumentwicklung und der Systeme in Deutschland, Frankreich und der Union beobachtet er eine sich verstetigende Annäherung hinsichtlich Raumvorstellung und Instrumentenauswahl.

Abschließend bietet Milstein im vierten Teil eine Zusammenfassung seiner Thesen. Das umfangreiche Quellenstudium findet Niederschlag in einem 132 Seiten starken Literaturverzeichnis. Das die Arbeit abrundende Sachverzeichnis erleichtert dem Leser, der sich nur mit Teilaspekten des Themenkomplexes befassen möchte, die Orientierung. Als besonderer Gewinn dieser Arbeit verbleibt für den Rezensenten, dass sie das Konzept der Territorialen Kohärenz durchgängig durch alle erörterten europäischen Politikbereiche vor dem Hintergrund der rechtsvergleichenden Untersuchung betrachtet und nicht zuletzt dadurch wertvolle Unterstützung leistet, sodass die vielfältigen offenen Fragen einer europäischen Raumentwicklung mit der notwendigen kritischen Distanz zum jeweiligen nationalstaatlich geprägten Vorverständnis diskutiert werden können. 